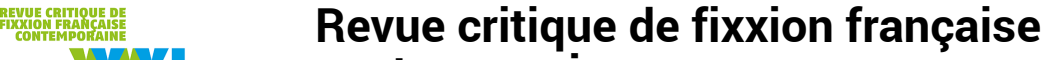 \\ XXI contemporaine
}

\section{Chaussure politique}

Les figures du pouvoir chez Nathalie Quintane (1996-2019)

\section{Marc-Antoine Blais}

\section{OpenEdition}

\section{Journals}

Édition électronique

URL : https://journals.openedition.org/fixxion/443

DOI : $10.4000 /$ fixxion. 443

ISSN : 2295-9106

Éditeur

Ghent University

Référence électronique

Marc-Antoine Blais, "Chaussure politique », Revue critique de fixxion française contemporaine [En ligne], 21 | 2020, mis en ligne le 15 décembre 2020, consulté le 17 février 2022. URL : http://

journals.openedition.org/fixxion/443; DOI : https://doi.org/10.4000/fixxion.443

Ce document a été généré automatiquement le 17 février 2022.

\section{cc) () $\odot$}

Les contenus de la Revue critique de fixxion française contemporaine sont mis à disposition selon les termes de la licence Creative Commons Attribution - Pas d'Utilisation Commerciale - Pas de Modification 4.0 International. 


\title{
Chaussure politique
}

Les figures du pouvoir chez Nathalie Quintane (1996-2019)

\author{
Marc-Antoine Blais
}

1 Le fait est désormais entendu : la production de Nathalie Quintane se clive entre deux parties distinctes. D'un côté, il y aurait les textes formalistes (intraitables) des débuts, qui s'étendraient des Remarques (1997) jusqu'à Grand ensemble (2008). De l'autre, il y aurait les textes politiques (plus aisément lisibles) de la maturité, inaugurés par un Tomates (2010) qui marquerait l'abandon par l'écrivaine de ses lubies avant-gardistes. Deux versants, deux pratiques, deux programmes, séparés par des cloisons étanches. À lire certains avis de la critique, tant médiatique qu'universitaire, on croirait qu'un paravent se dresse entre le premier et le second moments de l'œuvre, empêchant entre eux le moindre croisement, le moindre recoupement.

Ce partage, qu'atteste la réception récente des textes de l'écrivaine, apparaît d'autant plus convaincant qu'il rejoue un scénario connu, dans le champ littéraire français. Ce passage par Quintane "vers davantage de transitivité" Huppe, évoque en effet l'hypothèse de "retour" au réel que soutient le manifeste "Pour une littérature-monde en français" (2007) (à la suite, bien sûr, de Dominique Viart). Pendant des décennies, affirment ses cosignataires, "[l]e monde, le sujet, le sens, l'histoire, le référent [...] auront été mis entre parenthèses par les maîtres penseurs, inventeurs d'une littérature sans autre objet qu'elle-même". Nul "monde" sous la Terreur des textualistes, la production de ces dernierère's ne se référant plus, fatalement, qu'à ses propres structures. S'opposant à ce constat qui débouche, quelques lignes plus loin, sur la mise en parallèle du "reflux des avant-gardes propres aux années 1980 [...] avec l'effondrement du Mur de Berlin"3, Bruno Blanckeman souligne que "l'idée de retour bloque celle d'évolution" ${ }^{4}$. À trop caricaturer les positions avantgardistes, à trop insister sur l'épaisseur de la frontière "1980", on occulte forcément nombre d'expérimentations témoignant déjà, deux décennies auparavant, d'une volonté d'investir le monde ${ }^{5}$. Si le motif d'un retour au réel ne colle pas chez Quintane, il demeure néanmoins que l'ampleur de l'éclat suscité par la parution de Tomates participe de logiques non éloignées. Après une décennie d'atermoiements, l'écrivaine aborderait enfin de front la question du politique, transitant, selon Eric Lynch, "d'une 
écriture expérimentale qui était politique par le travail sur la langue qu'elle opérait, par exemple dans son livre Chaussure, à une littérature qui parle directement de politique"6. Exit l'"écriture expérimentale", bonjour la "littérature" : Quintane passerait d'un camp à l'autre, s'attachant aussi bien à confronter le pouvoir qu'à s'exprimer simplement.

3 Autant le préciser d'emblée: il y a fort à parier qu'aucun'e des chercheur e's convoquée's par cet article ne souscrit à l'hypothèse d'une Quintane bicéphale. En effet, c'est non pas à des lectures particulières, explicites et systématisées que je souhaite m'attaquer par ce qui suit, mais plutôt à l'ensemble de représentations qui sous-tend l'idée d'une partition dans l'œuvre de l'autrice. Ces représentations se manifestent dans la critique par des indices divers, actualisées tantôt sur le mode du discours, tantôt seulement par l'emploi d'un lexique révélateur. "Tournant", "bifurcation" (IV), "general shift" : la césure "2010" marquerait un changement de paradigme majeur dans la production de Quintane. Pourtant, l'écrivaine elle-même s'en défend :

Depuis quelques temps, je me suis remise à lire en public des extraits de mes premiers livres : Chaussure (P.O.L., 1997) ; Jeanne Darc (P.O.L., 1998). En effet, il n'y a pas, pour moi, de rupture entre ces débuts poétiques et une "renaissance" politique qui aurait commencé avec Tomates, en 2010, livre dont l'un des points de démarrage est l'affaire dite de Tarnac, ou encore Un œil en moins, paru en 2018, qui revient sur Nuit debout.9

Pas de "rupture" ni de "renaissance politique" : Quintane insiste sur le fil de continuité qui unirait ses premiers textes à ses parutions les plus récentes. Cet enjeu préoccupe visiblement l'autrice, qui accorde depuis Les années 10 (2014) un intérêt manifeste à la réception de ses ouvrages. Prenant acte de ce regard rétrospectif posé sur l'œuvre, j'interrogerai, dans la première partie de ma contribution, les propositions diverses soutenues tant par Quintane que par ses lecteur-rice's à l'égard de la division Chaussure/ Tomates, tâchant par là d'en problématiser les traits généraux. Puis, dans les deuxième et troisième parties, je montrerai que la question de la politique, loin de ne surgir dans l'œuvre qu'à partir de 2010, motive plusieurs des thèmes et des partis pris formels qui marquent sa "première partie". Représenter les figures du pouvoir, miner les imaginaires qui en fondent l'autorité, détourner leur discours: autant de procédés critiques qui caractérisent, depuis plus de vingt ans, la pratique de Quintane.

\section{Un tournant ? Quel tournant?}

Dans l'introduction de son article "L'insurrection qui vient par la forme" (2019) ${ }^{10}$, Huppe se penche sur les dynamiques nouvelles qu'instaurerait, chez Quintane, la parution de Tomates :

Au tournant des "années 10", comme elle les appelle, marquée par le durcissement du climat social et politique en France, Quintane se départit de l'idée, rétrospectivement jugée confortable, selon laquelle écrire de la poésie est un acte politique en soi. Désormais elle citera les noms des gouvernants, chroniquera l'actualité sociale et sortira de sa réserve de "fillette des classes moyennes qui comme un écrivain des colonies tâche d'écrire correctement en français ce qui ne lui plait pas". (IV)

6 Ces quelques lignes condensent plusieurs des enjeux que soulève la partition supposée de l'œuvre : aussi convient-il d'en déplier soigneusement les implications. En effet, il y a lieu de contextualiser la proposition voulant qu'“écrire de la poésie est un acte 
politique en soi". Ces mots font écho à un passage d'Un œil en moins (2018) (texte duquel provient aussi la citation finale du paragraphe) : "Avant (i.e. dans ma jeunesse), on pensait que faire de la poésie, peu importe laquelle, était un geste en soi politique. / Maintenant, ça ne suffit plus"11. De fait, c'est avant tout dans la "jeunesse" de Quintane que circulait cette conception, qui s'inscrit dans le sillon des théories de Tel Quel. Le fameux "la langue est fasciste" de Roland Barthes résume le cadre qui sous-tend un tel nouage entre écriture et politique, le travail poétique de la langue offrant, dans cette optique, des moyens de lutter contre sa charge idéologique intrinsèque. Si, d'une part, la Quintane des débuts reprend ces logiques par la "méfiance" plus marquée qu'elle manifeste "à l'égard du référent"12, comme le note Julien Lefort-Favreau, il ne m'apparaît pas superflu de souligner, d'autre part, qu'un écart non négligeable sépare sa poésie de celle des textualistes. "Ah" (1996), Chaussure et Grand ensemble (2008), loin de se contenter d'investir la langue des puissantees et d'en retourner les structures, figurent une série de gouvernantees, ne se gênent pas pour citer leurs noms. En ce sens, la politique de la littérature adoptée par Quintane avant la parution de Tomates ne se résume pas au parti pris de Tel Quel : l'écrivaine "parle" de politique depuis ses tout premiers écrits.

7 Les premières lignes d'“Astronomiques assertions" (2011) traitent sans détour de la question d'un changement de cap dans l'œuvre :

1. Mon amie A. P. (qui est mon amie moi-même) rechigne : pourquoi en es-tu venue à parler de Tarnac, alors qu'avant tu parlais de chaussure, et que tu parlais aussi bien de Tarnac en parlant de chaussure qu'en n'en parlant pas (de Tarnac) ?

1.1. En publiant Chaussure à la fin des années 1990, je n'ai pas publié Oiseaux dans le Ciel - puisque j'ai publié Chaussure.

1.2. Je me disais : chaussure, c'est Louboutin (à la fin des années 1990). Profitons de ce que chaussure soit Louboutin pour y glisser du tarnac (bien avant Tarnac même, car les poètes ont des visions un peu comme Nostradamus). ${ }^{13}$

Non, il ne s'agit pas pour la Quintane de 1997 d'écrire de la poésie sur des sujets sans conséquence et d'espérer, ce faisant, produire un effet politique (caricature qui ne saurait d'ailleurs dépeindre ni sa pratique, ni celle des Telquelien-ne-s). Au contraire, l'écrivaine affirme avoir "glissé du tarnac" dans ses premiers textes, c'est-à-dire qu'elle soutient que les thèmes, que les figures, que les procédés sollicités par ces derniers répondent, pour une part, à des motifs politiques. Est-il possible de "parler de Tarnac"14 tout "en parlant de chaussure" ? En d'autres termes : est-il possible de traiter d'enjeux politiques actuels sans s'y référer explicitement? Tel serait le pari de Quintane, qui s'afflige, dans "Pourquoi l'extrême gauche ne lit-elle pas de littérature ?" (2014), de ce qu'aux yeux de certain'e's, comme elle le constate à la sortie de Tomates, "pour parler de chômage dans un livre, il faut un chômeur, pour parler de dictature, il faut un dictateur" ${ }^{15}$. Dans un entretien accordé au magazine Diacritik en 2016, l'écrivaine étaye encore cette idée :

La critique se fait le plus souvent explicitement, sauf en littérature - en tout cas, telle que je la conçois et essaye de la "pratiquer". L'un des livres les plus ouvertement politiques serait, pour moi, l'Humble proposition pour empêcher les enfants des pauvres en Irlande d'être à la charge de leurs parents ou de leur pays, de Swift. Autant dire que ce n'est pas explicite, puisque ça passe par l'ironie et qu'il faut en déplier tout le feuilleté pour saisir ce qui se joue dans ce texte. / Des noms propres comme Tarnac ou Sarkozy fonctionnent comme des indices de ce que là où ils sont lus, c'est plus politique qu'avec, par exemple, Saint-Tropez ou Chaussure. Or, les lecteurs peuvent être légitimement déçus, puisque Tomates est tout aussi tarabiscoté que Saint-Tropez, dans un autre genre. Je ne sais pas si mes derniers 
livres sont - beaucoup - plus politiques que les précédents, mais ce qui est sûr,

c'est que l'époque que nous vivons tous nous force à l'être. ${ }^{16}$

Des Remarques aux parutions les plus récentes, la critique, chez Quintane, serait toujours implicite. Des textes considérés "plus politiques" comme Tomates, s'ils citent des "noms propres" et s'il esquissent les traits d'une situation aisément reconnaissable, ont peu à voir en effet avec le mode d'engagement littéraire que défendaient, à une autre époque, certain'e's écrivain'e's communistes. C'est qu'il s'agit moins pour l'autrice de défendre une thèse ou de gloser sur la teneur problématique de faits sociaux que de détricoter, par un travail de scrutation, les lieux communs qui soutiennent divers appareils de domination. Certes, ce travail s'attaque à des objets sensiblement différents d'un ouvrage à l'autre, la commune de "Tarnac" apparaissant a priori plus chargée politiquement que celle de "Saint-Tropez", par exemple. Pourtant, non seulement Tomates et Saint-Tropez (2001) traitent-ils, par le truchement de ces deux lieux, d'enjeux incontestablement politiques, mais encore s'y prennent-ils de façons tout à fait similaires. Dans un cas comme dans l'autre, c'est bien de la critique implicite d'un imaginaire (policier, touristique) qu'il s'agit, et c'est par une construction "tarabiscotée" que des représentations communes sont problématisées.

Alors, à quoi tient l'idée d'une partition dans l'œuvre de Quintane? Quelle pratiques radicalement nouvelles Tomates inaugure-t-il donc? Le fait de parler de politique? Non, la proposition ne tient pas la route. L'emploi d'une instance d'énonciation subjective? Non, bien sûr : l'écrivaine use du procédé depuis Remarques jusqu'à Grand ensemble, en passant par Début (1999) (sous-titré "Autobiographie"). Le fait de "cit[er] les noms des gouvernants, [de] chroniqu[er] l'actualité sociale", comme l'avance Huppe? L'observation est plus intéressante : si on retient de cette affirmation la question de la "chronique", il y a lieu de reconnaître qu'elle n'est pas sans fondements. En effet, avant 2010, Quintane se préoccupe surtout de "repasser l'Histoire" ${ }^{17}$, comme le formule joliment Alain Farah, les moments les plus explicitement politiques de l'œuvre traitant de la signature de l'armistice de 1918 (dans Cavale ${ }^{18}$ ) ou de la guerre d'Algérie (dans Grand ensemble), mais rarement de situations actuelles. Ce transit d'un traitement de l'Histoire à celui de l'actualité sociale saurait soutenir l'idée d'un changement chez Quintane: l'écrivaine désigne bel et bien, à partir de 2010, des enjeux politiques contemporains au présent de l'écriture. Pour autant, je l'ai souligné, l'hypothèse selon laquelle l'irruption du nom "Sarkozy" marquerait un basculement dans l'œuvre n'est pas des mieux venues: les politicien'ne's peuplent les textes de Quintane depuis les années 1990. Des Mao, Pétain et Mussolini de "Ah" à la Marine Le Pen de "Stand up" (2014) et des Kroutchev et Imelda Marcos de Chaussure aux Hollande et "Manu" Macron d'Un œil en moins, les personnalités politiques sont légion chez l'écrivaine. De fait, l'une des façons les plus efficaces de mettre au jour la cohérence de la production de Quintane serait, davantage encore que par l'analyse de son travail sur l'infrapolitique ${ }^{19}$, de souligner la récurrence, en son sein, de ces figures du pouvoir. Manifestations immédiatement saisissables du souci politique qui sous-tend l'œuvre, les politicien-e's ventriloquée's par Quintane illustrent exemplairement les modes par lesquels sa critique implicite s'attaque, morceau par morceau, aux imaginaires du pouvoir. Parfois, pour parler de dictature, "un dictateur" n'est pas de trop. 


\section{Fendre le bloc}

11 La question de l'ironie est incontournable pour qui s'intéresse à la pratique de Quintane, qui ne retient pas, de Swift, que le penchant pour la critique. La lecture des portraits de gouvernante's composés par l'écrivaine ne manque pas, en effet, de provoquer le rire (parfois même carnavalesque): aussi convient-il à présent de se pencher brièvement sur les modes par lesquels Quintane reprend à son compte ce procédé. L'ironie swiftienne se caractériserait en partie, selon David Ball, par un travail $\mathrm{du}$ "contraste entre l'hypocrisie et la vanité de l'homme d'une part, et sa condition matérielle, médiocre et méprisable, de l'autre" ${ }^{20}$. Du Tale of a Tub (1704) aux Gulliver's Travels (1726), ce serait avant tout par un "démenti physique ${ }^{21}$ " que Swift ferait chanceler la prestance de ses personnages, qu'il opposerait à leurs affirmations des négations implacables. Cette méthode n'est pas étrangère à Quintane, qui imagine Pétain la morve au nez dans "Ah" et qui figure Macron ou bien aux courses, ou bien un œuf au front dans Un œil en moins. Pourtant, si l'ironie de l'écrivaine a beaucoup à voir avec celle de Swift, elle ne saurait se résumer à un tel jeu d'antinomie, ainsi que le note Benoît Auclerc :

L'ironie du oui et non telle que la pratique Quintane s'oppose [...] dans une large mesure aux formes plus communément répertoriées de l'ironie - ironie corrective, maniant la louange pour le blâme, tournant en dérision les objets auxquels elle s'attaque, "antagoniste" au "discours sérieux", qui se laisse relativement facilement enclore dans une figure (l'antiphrase), avance des énoncés à même de faire sourire un lecteur complice qui les annulera pour (r)établir le "bon sens". ${ }^{22}$

12 Ce parti pris du "oui et non", formule qu'Auclerc emprunte à Pierre Schoentjes, exclut tout jugement univoque: "si cela peut s'avérer ponctuellement efficace, la question n'est pas de simplement retourner un discours adverse (logique antiphrastique de l'ironie corrective), mais de souligner la porosité des positions et des discours" (SI 54). Peu d'ironie au sens rhétorique du terme, chez Quintane, mais plutôt un souci de rapprocher, par divers moyens, des situations que tout éloigne a priori : il s'agit pour l'écrivaine de forcer des rencontres, de favoriser des chocs.

Dans une "vidéolecture" d'Un œil en moins diffusée par P.O.L, Quintane affirme ne pas pouvoir écrire sans "qu'il y ait une sorte d'humour, qui est dans la syntaxe même, qui n'est pas une volonté de faire gag, mais qui va avec l'idée de faire attention au plus petit détail"23. "Faire attention au plus petit détail" : la formule souligne non seulement un ressort humoristique central chez l'écrivaine, mais aussi l'un des traits fondamentaux de son œuvre entière. Depuis ses Remarques qui scrutent le banal et le quotidien jusqu'à son Les enfants vont bien (2019) qui offre une vue rapprochée de discours divers, Quintane s'adonne à un travail du détail, elle s'attache à révéler de l'inaperçu. Le cas Saint-Tropez est, à mon sens, particulièrement éclairant pour comprendre les enjeux qui motivent cette pratique. Avançant que la station balnéaire "est pareille, quand on y pense, à un grand bloc insécable" 24 fait de représentations collectives, Quintane se demande, dans les dernières pages du texte, "[q]uel élément hétérogène pourrait fendre ce bloc"25. Si l'autrice reproduit dans l'ouvrage une série de cartes postales ou si elle énumère des noms de célébrités associés à la ville, c'est donc avant tout pour parvenir à identifier "quel élément hétérogène" (par exemple la torpille, spécialité locale de la commune selon Quintane ${ }^{26}$ ) saurait fendre le bloc "Saint-Tropez", c'est-àdire problématiser les lieux communs qui lui collent. Les caricatures de politicien'ne's qui jalonnent l'œuvre participent de logiques tout à fait similaires. En effet, lorsqu'elle 
brosse le portrait de figures du pouvoir, Quintane entend moins retourner un bloc de représentations communes qu'y insérer de l'équivoque, qu'en mettre au jour la face cachée. Auclerc le souligne : pour l'écrivaine, "ironiser consiste à tenir compte de ces réalités apparemment incompatibles et qui pourtant coexistent, à maintenir ensemble ce qui paraît contraire et opposé" (SI 48-49). Si ces latences exhibées suscitent parfois le rire par le "oui et non" qu'implique leur incompatibilité avec l'ethos politicien, ce rire n'est cependant soutenu par aucune "identité rhétorique" ${ }^{27}$, par aucun programme clair. Fendre le bloc, oui, mais pas sous l'impulsion d'une ironie corrective : plutôt par un jeu de loupe qui révèle, un détail à la fois, quelques failles des imaginaires qui soutiennent Marine Le Pen, Macron, Pétain ou Kroutchev.

"Stand up. (une visite de Marine Le Pen en province)" est le premier des neufs textes qui composent Les années 10 (2014). Le sous-titre ne trompe pas: il s'agit bel et bien d'une tentative par Quintane d'"imaginer [une] visite de Marine Le Pen dans la ville de D." (LA 38) (que l'on devine être Digne-les-Bains, commune où réside l'autrice). Pendant une trentaine de pages, l'écrivaine décrit la tournée de commerces qu'entreprend la présidente du Front national ${ }^{28}$, détaillant l'itinéraire de la femme politique et croisant, au détour de certains passages, sa propre perspective à la sienne. Les premières lignes donnent le ton, Quintane se représentant une incursion (littérale) de Le Pen dans sa tête :

Marine Le Pen est au plafond, elle s'y installe, elle s'y est aménagé une petite chambre avec un lit à une place, un fauteuil crapaud, une table de chevet où repose une Vie de Georges Pompidou, elle a changé le lustre qui était un peu vieillot, avec ses tulipes, elle a placé un néon rose en forme de toucan sur un mur et elle déguste un Twix en inspectant ses ongles de doigts de pied vernis[.] (LA 7)

De la Vie du gaulliste Georges Pompidou au fauteuil crapaud, et du lustre remplacé parce que trop "vieillot" au néon rose, le portrait est déjà éloquent : le-la lecteurrice reconnaît la Le Pen de la dédiabolisation, celle qui conserve pour l'essentiel les principes admis du Front national, mais qui s'attache à en renouveler et à en moderniser l'image. L'aspect fantaisiste de cet exemple se transpose dans l'ensemble du texte, qui liste au fil des pages une série d'inclinations supposées de la femme politique : elle "préfère les chocolats noirs" ( $L A$ 9), elle "aime beaucoup les petites pensions de famille" (LA 15), elle termine ses soirées avec "une bonne clope et un café" (LA 15), elle apprécie les bars "où l'on joue en terrasse à la pétanque en écoutant de la musique de bikers" (LA 22), etc. Ces conjectures, qui résonnent avec l'ethos de Le Pen, font sourire tantôt par leur caractère anodin, tantôt par leur caractère burlesque, tantôt par la précision des traits de personnalité qu'elles impliquent. D'autres passages caricaturent des traits physiques de la femme politique, qu'il s'agisse de la vigueur qu'elle dégage ("elle s'en retourne, envoie un coup de godasse dans la porte qui coince", LA 22) ou de la raucité de sa voix (elle "[emplit] l'espace de sa belle voix grave éraillée de bluesman, et elle en pousse une petite, justement, elle entonne un air de cuisine", LA 11). Il s'agit, ce faisant, d'inventorier les composantes du dispositif "Marine Le Pen", moins pour y opposer une critique explicite ${ }^{29}$ que pour prendre la mesure de l'imaginaire qui sous-tend ses propositions politiques, pour saisir les termes de la conjoncture qui favorise ses succès électoraux (selon une note du texte, la liste du Front national récolte $33,39 \%$ des suffrages dans la ville de D., lors des élections municipales de mars 2014). Au cours de son "stand up" livré devant les commerçante-s, Le Pen évoque une "ex-socialiste qui n'a rien trouvé de mieux à dire pour expliquer son passage du PS au FN qu'enfin elle s'était sentie en famille, dans une ambiance familiale, le 
PS était une famille froide, c'est-à-dire pas du tout une famille, le FN une famille chaude : une famille." (LA 28-29) La familiarité de la fille de Jean-Marie Le Pen et de la tante de Marion Maréchal favoriserait significativement l'adhésion de l'électorat à son discours, Marine Le Pen ayant compris que "les choix politiques, c'est pas une histoire de logique" (LA 27). Ce constat justifie, d'ailleurs, jusqu'au texte lui-même : si la critique explicite de propositions raisonnées a sa place en terrain politique, la mise au jour des mythes socialement actifs qui les supportent est, nous rappelle Quintane, tout autant incontournable.

Des logiques du même ordre motivent, dans Un œil en moins, la série de descriptions de celui que Quintane surnomme "Manu" Macron, figure centrale de l'ouvrage. Dans le passage suivant, c'est non pas dans sa tête, mais au supermarché que l'écrivaine se représente l'homme politique :

Je faisais mes courses avec Manu Macron à l'Inter, et c'est là qu'on s'est engueulés parce qu'il voulait que je prenne des panais bios, et moi je lui expliquais que le bio pour les panais, c'était ridicule, mais j'étais incapable de développer la raison pour laquelle c'était ridicule, en fait, je bloquais à chaque mot, et lui a fini par mettre les panais d'autorité dans le caddie; on ne voyait plus que ces sortes de carottes plissées dans leur emballage bistre[.] (UO 331)

Ici encore, le texte souligne un trait caractéristique d'un dirigeant: la résolution inébranlable de Macron, son "autorité". Le président jupitérien, qui n'est pas reconnu pour son sens du compromis, ira d'ailleurs jusqu'à affirmer dans un entretien avec la Nouvelle revue française qu'il "[assume] la part parfois univoque, unilatérale de toute décision"30. Sûr de ses moyens, Macron n'a pas "la démarche craintive et tressautante de Sarkozy" (UO 332), avance Quintane, "[q]uelque chose de la lumière" (UO 332) émane de lui et force l'admiration de l'électorat. C'est pour désamorcer cette superbe que l'autrice délocalise le président du palais de l'Élysée pour le camper dans un Intermarché, sa fermeté ne manquant pas, dans ce nouveau système de référence, de tourner au risible. En outre, Quintane se figure elle-même aux côtés de "Manu" Macron, le pare d'un sobriquet, se dispute avec lui: sur ce plan encore, la verticalité que revendique l'homme politique ne tient plus, il quitte les hauteurs de la fonction présidentielle pour se mêler au commun. Pour autant, c'est moins par le renversement carnavalesque opéré que ce court portrait agit politiquement que par son travail de détricotage d'un ethos. Transposée dans un supermarché, privée de l'environnement symbolique où s'exerce son pouvoir, la "lumière" que diffuse le président s'estompe : de l'aura jupitérienne ne subsiste qu'un autoritarisme ridicule, car dépouillé du décor qui en assure ailleurs le poids.

Cette étude des gestes et des manières d'être des gouvernant·es, loin de ne caractériser que la dernière décennie de l'œuvre, a cours dès les tout premiers textes de Quintane. "Ah", publié dans le deuxième numéro de la Revue de littérature générale, parait un an avant Remarques et Chaussure. L'écrivaine s'y penche sur le quotidien et sur la pratique du pouvoir de trois hommes politiques: Mao, Pétain et Mussolini. Dans le deuxième tableau du texte, un narrateur inconnu jauge les dispositions de pensée qui conviennent à un chef d'État: "Tandis que je ne pense à rien, une guerre est en train de se déclencher sur le front de l'Est. Donc, mieux vaut pour moi penser à en déclencher une à l'Est, par exemple" ${ }^{31}$. L'homme politique en conclut : "L'idéal serait que je puisse penser longtemps, et continûment: moins il y a de trous, moins je laisse à d'autres l'envie et l'occasion de les combler, en y logeant leur propre décision." ( $\left.A \mathrm{n}^{\circ} 2\right)$ Cette utopie du commandement implique une attention permanente, une vue globale sur les 
affaires de l'État qui ne recèlerait aucune faiblesse, qui ne se laisserait miner par aucune influence extérieure. C'est l'impression qu'offrent notamment les affiches de propagande du régime de Vichy, qui présentent un maréchal Pétain au regard droit, à la moustache savamment ciselée, incarnant l'État par sa seule personne. Or Quintane s'interroge, dans le cinquième tableau de "Ah": comment l'imperturbabilité des puissant-ess peut-elle tenir face aux contingences de la vie quotidienne? "Combien de fois Philippe Pétain / fut-il enrhumé, de 1940 à 1944? / D’être enrhumé, / quelles réactions furent les siennes? / Du fait que quelque chose / coule / de soi / sans contrôle, / Qu'est-ce qui s'exerce, / se substituant aux réponses classiques?" ( $\left.A \mathrm{n}^{\circ}{ }^{5}\right)$ Le rhume, défaut négligeable, voire risible, mais sur lequel Pétain ne peut espérer exercer de contrôle, perce d'un "trou" l'image communément partagée du maréchal, offrant par là même une prise sur elle. Quintane emploie un procédé similaire dans Chaussure ${ }^{32}$, dans la page qu'elle consacre à l'histoire de "la chaussure de Kroutchev". Le président de l'URSS apparait à l'ONU, "assis à une table. Il avait pour ainsi dire l'allure d'un homme important, son air. Aussi était-il, en effet, un homme important" ${ }^{33}$. Puis, Kroutchev se saisit d'une chaussure, qu'il frappe sur la table ${ }^{34}$. Dès lors, l'homme politique disparaît :

Non qu'il ait effectivement disparu, la chaussure ayant pris, par son élévation à

table, des proportions hors normes, mais, aux yeux du spectateur, son air, lui, avait

bel et bien disparu, brouillé par le surgissement impromptu d'un objet certes banal,

mais déplacé. ${ }^{35}$

À quoi tient l'"air" d'un homme important, nous demande Quintane ? Par ce rappel de l'anecdote de "la chaussure de Kroutchev", l'écrivaine insiste, une fois de plus, sur la valeur politique des détails. Si c'est par une accumulation de petites choses que se coagule un "air" (par "[u]ne lueur (un éclat) dans l'œil (le regard)" ou par "[d]eux épaules impassibles" ${ }^{36}$ ), Quintane souligne que de petites choses, inversement, peuvent aussi bien le fracturer. Rhume ou chaussure, les visées qui motivent l'insertion d'éléments hétérogènes dans les portraits de figures du pouvoir sont coup sur coup les mêmes : fendre l'ethos que se composent les politicien'ne-s, ouvrir un espace critique en son sein.

"Comment ne pas voir le ridicule des puissants?" (UP). La question, lancée par Quintane dans un entretien accordé au Monde en 2018, souligne la nécessité des rapports qu'entretiennent, dans ses ouvrages, humour et politique : impossible pour l'écrivaine de critiquer le pouvoir sans ironiser, sans se moquer de ses représentante-s. Dans les portraits de politicien'ess plus qu'ailleurs dans l'œuvre, une identité rhétorique

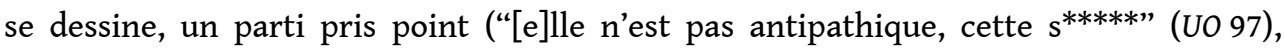
affirme Quintane à propos d'Hollande): pourtant, ce qui sous-tend l'exhibition du ridicule des puissantees ou le grossissement de leurs traits, ce n'est jamais tant une volonté de corriger qu'un souci de faire saillir. De Chaussure à Un ceil en moins, de Kroutchev à Macron, Quintane s'attache à révéler, par son ironie du "oui et non", les coulisses de jeux de représentations qui fortifient, au quotidien, l'ascendant des gouvernant·e's.

\section{Pousser, détourner}

En terrain avant-gardiste, les innovations formelles sont rarement dissociables des innovations politiques qu'elles promettent. Ainsi en est-il du détournement des situationnistes, que Guy-Ernest Debord et que Gil J. Wolman conçoivent, dans leur 
entreprise de systématisation du procédé, comme "la première ébauche d'un communisme littéraire" ${ }^{37}$. Cette forme de "propagande éducative" ${ }^{38}$ permettrait, à leurs yeux, de "[battre] en brèche toutes les murailles de Chine de l'intelligence" ${ }^{39}$ par un travail de ponction et de subversion de discours (voire potentiellement de tout élément, “pris n'importe où" ${ }^{40}$ ). Si elle laisse à d'autres la production de propagande, Quintane ne détourne pas moins nombre de formules circulant au sein de l'espace social dans ses textes, depuis Saint-Tropez jusqu'à Les enfants vont bien, décrit à juste titre comme "un livre de montage" ${ }^{41}$. Dans ce dernier ouvrage, qui fixe la rumeur collective à partir de laquelle "est fabriqué, puis justifié et légitimé" (EB 7) le sort des réfugiée's en France, l'écrivaine reproduit tels quels une série de fragments textuels accolés les uns aux autres, qu'il s'agisse d'extraits de la presse, de messages échangés dans des réseaux d'aide... ou de déclarations de politicien.ne's. De fait, non seulement la construction "tarabiscotée" de Les enfants vont bien compose-t-elle un panorama varié des phraséologies qui forment le discours social, mais encore offre-t-elle un point de vue privilégié sur la grammaire du pouvoir, qu'elle recadre et qu'elle investit de sens nouveaux. Si scruter les gestes et les pratiques des politicien.ne's est un moyen efficace de fragiliser leur stature, détourner le discours des gouvernante-s demeure utile pour qui veut critiquer l'ensemble des dispositifs qui en assoient le pouvoir.

Aussi fascinant que soit le montage, c'est un détournement d'un autre ordre qui m'intéresse, chez Quintane. Dans "Pourquoi l'extrême-gauche ne lit-elle pas de littérature?", dernier texte de Les années 10, l'écrivaine consacre quelques pages aux entreprises de "traduction" de Stéphane Bérard : par sa réécriture du premier tome des Mémoires d'espoir (1970) de Charles de Gaulle, le poète ferait "revenir (l'insu de) [son] ridicule, comme on frit au ralenti un oignon pour mieux en révéler les saveurs" (LA 181) ; par son remaniement du Mein Kampf (1925) d'Adolf Hitler, il ferait le pari de "travailler le texte - de mettre ce texte au travail - pour que, dans le détail, sa folie soit révélée" (LA 183). Si Quintane convient "qu'historiquement les personnes de Gaulle et Hitler ne sont aucunement comparables" (LA 182), dans l'un et l'autre de ces cas, il s'agit pourtant pour Bérard de "[f]aire naître, ou pousser poétiquement, l'autre abruti du texte dans le cours de sa réécriture" (LA 183). Plutôt que de laisser les ouvrages intouchés et de se contenter d'en réagencer les morceaux (ou encore, comme pour la plupart des rééditions actuelles de Mein Kampf, d'ajouter au livre un appareil critique), le poète entend détourner le discours des puissantees de l'intérieur, "pousser poétiquement" le ridicule ou la folie qu'il recèlerait. C'est une stratégie que Quintane elle-même adopte, à divers degrés, dans plusieurs de ses textes. Dans Tomates, par exemple, elle reprend des propos tenus par la femme politique française Michèle AlliotMarie, l'une des figures centrales de l'affaire de Tarnac: “Je revenais de T., j'écoutais Europe 1, Michèle Alliot-Marie parlant de son passage de l'Intérieur à la Justice, de ce que la sécurité n'était pas seulement l'affaire de la police mais de l'Éducation nationale, des commerçants, des familles, etc." (T 68-69). Puis, dans les lignes qui suivent, Quintane se demande si la proposition "la sécurité est l'affaire de tous" signifie qu'elle "[s]'occupe aussi de sécurité" :

je vérifie si le gaz est bien fermé après l'avoir fermé, je ne marche pas sur le savon quand je suis dans la baignoire, je tourne deux fois la clé dans la serrure contre les cambrioleurs, je m'aplatis sur le sol s'il y a de la fumée. Est-ce que Michèle AlliotMarie veut bien dire cela - que je suis aplatie sur le sol, que je ne glisse pas dans ma baignoire, que je n'explose pas car il y a trop de gaz - quand elle parle de sécurité ? (T 69) 

s'attache à en imaginer la synthèse :

Sécurité domestique = sécurité publique $:$ je m'aplatis s'il y a de la fumée, je contourne le mendiant (pour ne pas favoriser l'aumône), j'ôte le savon du dedans de la baignoire et je le pose sur son bord, je complète les fiches d'identification des enfants en bas âge, je ferme le gaz, je signale le sans-papiers à la préfecture, je mets un trois-points à ma porte d'entrée, etc. ( $T$ 70)

Ainsi, c'est à la fois par la monstration de la double acception du terme "sécurité" (qui, dans la grammaire du pouvoir, désigne autant la protection de l'intégrité physique des citoyen-ne's que le maintien de l'ordre par la police) et par un raisonnement par l'absurde que Quintane souligne le ridicule de la proposition de la ministre. Paraphraser la rhétorique d'Alliot-Marie, en "pousser" les logiques sous-jacentes : telles sont les tactiques sollicitées pour détourner le discours de la femme politique.

Ce travail d'énoncés portés par les figures du pouvoir s'ancre aussi dans la "première partie" de l'œuvre de Quintane. Juste avant qu'elle se représente, dans "Ah", un Pétain enrhumé, l'écrivaine "traduit" en effet un paragraphe entier des Citations du président Mao Tse-Toung (1966) (mieux connues en français sous le nom du Petit livre rouge). "Nous devrions apprendre à jouer correctement du piano. Peut-on dire que celui qui joue mal du piano en joue réellement ? [...] [I]l faut connaître l'instrument, pour ainsi dire, de l'intérieur ; l'ouvrir, tâter et frapper les cordes, faire claquer le couvercle, grimper dessus !" $\left(A n^{\circ} 4\right)$ Ces extraits de la réécriture de Quintane font écho aux phrases qui concluent le passage correspondant, dans les Citations:

nous ne devons pas réserver notre attention à quelques problèmes seulement, à l'exclusion des autres. Partout où il y a un problème ; il faut frapper la touche [...]. Certains jouent bien du piano, d'autres mal, et la différence est grande entre les mélodies qu'ils en tirent. Les camarades des comités du Parti doivent apprendre à bien "jouer du piano". 42

Quintane radicalise (et ridiculise) les propositions de Mao : chez elle, non seulement convient-il de distinguer celui qui joue mal du piano de celui qui en joue bien, mais encore est-il permis de douter que le premier en joue même "réellement"; non seulement convient-il de "frapper" des touches, mais encore faut-il "ouvrir" l'instrument, "faire claquer" son couvercle. Par la déclinaison d'une série de remarques et d'injonctions à l'incongruité croissante, Quintane actualise quelques-unes des virtualités que renferme la métaphore musicale de Mao, rendant mieux visible, de fait, la folie de la rhétorique autoritariste qui la sous-tend. Ce jeu critique se poursuit douze ans plus tard dans Grand ensemble, où l'écrivaine met en pratique deux slogans répétés par les dirigeants français à l'époque de la guerre d'Algérie :

par tous les moyens / ou / on a retiré les gants / enregistre aussi bien le poulet partant dératé avec toutes ses plumes, gagnant la campagne [...] / ou / la section menue de l'animal en os eux-mêmes hachés, la dispersion des cendres méconnaissables aux quatre coins, la liquidation des plaques des numéros des poulets, dont on fond le métal pour fabriquer des marmites. / Que par tous les moyens ne change pas l'État démocratique en dur confond les poulets. ${ }^{43}$

Comme le note Lynch: "It is partly these phrases [on utilisera tous les moyens et on ne mettra pas les gants] functioning performatively that led soldiers to enact the brutalities of the war" (UV 193). Par là, illustrer les modes d'application potentiels de ces deux formules est une façon pour Quintane de révéler l'extrême brutalité qu'elles autorisent: "par tous les moyens" appartient moins au registre discursif d'un État 
démocratique qu'à celui d'un État totalitaire (susceptible de hacher, de liquider). Si, comme l'avance l'écrivaine, "[u]n type de phrase correspon[d] à un type d'État" (GE 102), opposer au discours des puissantees une "pratique de la langue" (GE quatrième de couverture) capable d'en faire surgir les latences permet d'exhiber non seulement la face cachée de la grammaire du pouvoir, mais aussi celle des institutions que consolident son lexique, sa syntaxe.

Par le travail de l'un de ses motifs particuliers, celui des figures du pouvoir et de leur discours, j'ai exposé la grande cohérence de la production de Quintane. À moins de ne retenir de Chaussure qu'un penchant pour l'exploration formelle, à moins de voiler la portée sociale (voire la "transitivité") des premiers textes de l'œuvre, il m'apparaît en effet difficile de soutenir que Tomates marquerait, chez l'écrivaine, un éveil politique tardif. Quintane porte, au fil des décennies, une attention constante aux mythes qui organisent la pensée collective: d'une station balnéaire ou d'un'e gouvernant·e, elle retient avant tout des blocs (vite fendus) de représentations communément partagées. Oui, de la première au second, un pas est franchi : le-la politicien.ne, incarnation par excellence de l'État, met en jeu des rapports de pouvoir plus saillants que ceux que soulève une ville touristique. Ce pas, pour autant, n'est pas un saut. Loin de scruter d'un côté Kroutchev, de l'autre les Louboutin, Quintane offre par ses textes une vue synoptique de phénomènes de pouvoir de degrés divers, qu'elle s'attache à penser conjointement. Dans Grand ensemble, le détournement de slogans de gouvernant·e's suit la mise au jour du colonialisme latent de dispositifs de commémoration (GE 51-75) ${ }^{44}$; dans Les enfants vont bien, "le cynisme et l'opportunisme sans frein des hommes politiques" (EB 8) voisinent "la gestion administrative à la fois débonnaire et implacablement bureaucratique des centres d'accueil [pour réfugiés]" (EB 8). Il s'agit par là de multiplier les amorces, de s'attaquer au pouvoir sur différents fronts, de ne négliger ni ses manifestations les plus courantes, ni ses formes les plus insidieuses ou les plus occultées. La Marine Le Pen la plus dangereuse n'est peut-être pas celle qui arpente les rues, mais celle, plus petite, qui s'infiltre dans les têtes.

\section{NOTES DE FIN}

1. Justine Huppe, "L'insurrection qui vient par la forme", COnTEXTES, $\mathrm{n}^{\circ}$ 22, "La fiction contemporaine face à ses pouvoirs", 2019, URL: https://journals.openedition.org/contextes/ 6975 , consulté le 31 mai 2020 ; dorénavant IV.

2. Muriel Barbery et al., "Pour une littérature-monde en français", Le Monde, 15 mars 2007, URL : https://www.lemonde.fr/livres/article/2007/03/15/des-ecrivains-plaident-pour-un-roman-enfrancais-ouvert-sur-le-monde_883572_3260.html (consulté le 31 mai 2020).

3. "Roland Barthes égale Joseph Staline, CQFD", complète-t-il. Bruno Blanckeman, "Objectif: réel", dans Barbara Havercroft, Pascal Michelucci, Pascal Riendeau (dirs), Le roman français de l'extrême contemporain. Écritures, engagements, énonciations, Montréal, Nota bene, 2010, <Contemporanéités>, p. 226-227.

4. Ibid., p. 228. 
5. Si je me distancie, sur ce point spécifique, des propositions du manifeste, il m'apparaît important de reconnaître ici la justesse de sa critique de la relation centre-périphérie qui régit le champ littéraire français.

6. Eric Lynch, "Nathalie Quintane : Nous, le peuple", Marges, n 21, "Manifestes", 2015, p. 96.

7. Nicolas Truong, "Nathalie Quintane: Une partie de l'extrême gauche lit davantage de littérature”, Le Monde, 14 mars 2018, URL : https://www.lemonde.fr/idees/article/2018/03/14/ nathalie-quintane-une-partie-de-l-extreme-gauche-lit-davantage-de-

litterature_5270608_3232.html (consulté le 31 mai 2020) ; dorénavant UP.

8. Eric Lynch, Unidentified Verbal Objects: Contemporary French Poetry, Intermedia, and Narrative, thèse de doctorat, Graduate Faculty in French, City University of New York, 2016, f. 182 ; dorénavant UV.

9. École supérieure d'art Pays Basque, "Nathalie Quintane. Conférence", Centre national des arts plastiques, 2 mars 2020, URL: https://www.cnap.fr/nathalie-quintane-0 (consulté le 31 mai 2020).

10. Cet article est par ailleurs passionnant : je ne peux qu'y renvoyer.

11. Nathalie Quintane, Un œil en moins, P.O.L, 2018, p. 231; dorénavant UO.

12. Julien Lefort-Favreau, "Défaire les narrations, renverser l'hégémonie : pratiques de la liste chez Nathalie Quintane”, dans Nathalie Dupont et Éric Trudel (dirs), Poétiques de la liste et imaginaire sériel dans les lettres (XX et $\mathrm{XXI}^{e}$ siècles), Montréal, Nota bene, 2019, <Grise>, p. 212 ; dorénavant $D N$.

13. Nathalie Quintane, “Astronomiques assertions", dans Jean-Christophe Bailly et al., "Toi aussi, tu as des armes: poésie \& politique”, Paris, La Fabrique, 2011, p. 175.

14. L'affaire de Tarnac est amorcée en 2008 par l'arrestation de neuf militant·e-s (dont une partie vivait dans le village du même nom) soupçonnée's d'avoir saboté des caténaires.

15. Nathalie Quintane, Les années 10, Paris, La Fabrique, 2014, p. 198; dorénavant LA.

16. Emmanuelle Jawad, "Nathalie Quintane : l'espoir d'être un peu moins asservis (Création et politique 2)", Diacritik, 27 juillet 2016, URL : https://diacritik.com/2016/07/27/nathalie-quintanelespoir-detre-un-peu-moins-asservis-creation-et-politique-2/ (consulté le 31 mai 2020).

17. Alain Farah, Le gala des incomparables. Invention et résistance chez Olivier Cadiot et Nathalie Quintane, Paris, Classiques Garnier, 2013, <Études de littérature des Xxe et XXI ${ }^{\mathrm{e}}$ siècles>, p. 191 ; dorénavant GI.

18. Nathalie Quintane, Cavale, Paris, P.O.L, 2006, p. 141-154. À ce sujet, voir GI 212-213.

19. Alain Farah mentionne ce concept (emprunté à Charlotte Nordmann) dans GI 227.

20. David Ball, "Vers une théorie de l'ironie : perspectives sur Swift", Études anglaises, vol. 29, nº 1, janvier-mars 1976, p. 14.

21. Ibid.

22. Benoît Auclerc, "Prendre au sérieux (sur l'ironie)", dans Benoît Auclerc (dir.), Nathalie Quintane, Paris, Classiques Garnier, 2015, <Écrivains francophones d'aujourd'hui>, p. 50 ; dorénavant SI.

23. Jean-Paul Hirsch, "Nathalie Quintane Un œil en moins", YouTube, 29 mai 2018, URL : https:// www.youtube.com/watch?v=0Tpnao3TgTQ (consulté le 31 mai 2020).

24. Nathalie Quintane, Saint-Tropez - Une américaine, Paris, P.O.L, 2001, p. 77.

25. Ibid.

26. Ibid., p. 27.

27. Nathalie Quintane, Tomates, Paris, 2010, p. 52 ; dorénavant T.

28. Aujourd'hui nommé Rassemblement national.

29. Sinon par la description, ajoutée en fin de texte, d'un rassemblement organisé pour s'opposer à la venue de Le Pen. 
30. Cité par L'Obs, “Emmanuel Macron : J'assume la verticalité du pouvoir”, L'Obs, 27 avril 2018, URL : https://www.nouvelobs.com/politique/20180427.OBS5897/emmanuel-macron-j-assume-laverticalite-du-pouvoir.html (consulté le 31 mai 2020).

31. Nathalie Quintane, "Ah", "no 2", Revue de littérature générale, Paris, P.O.L, vol. 96, n², "Digest", 1996, section 33; dorénavant A.

32. Au sujet des trois pages de Chaussure consacrées à Kroutchev et à Imelda Marcos, voir GI 191-194.

33. Nathalie Quintane, Chaussure, Paris, P.O.L, 1997, p. 84.

34. La véracité historique de cette anecdote, qui aurait eu lieu en 1960, est aujourd'hui contestée.

35. Ibid.

36. Ibid.

37. Guy-Ernest Debord et Gil. J. Wolan, "Mode d'emploi du détournement", Les lèvres nues, $\mathrm{n}^{\circ} 8$, mai 1956, p. 5.

38. Ibid., p. 2.

39. Ibid., p. 5.

40. Ibid., p. 2.

41. Nathalie Quintane, Les enfants vont bien, Paris, P.O.L, 2019, p. 7; dorénavant EB.

42. Mao Tse-Toung, Citations du président Mao Tse-Toung, Pékin, Éditions en langues étrangères, 1967 [1966], p. 125.

43. Nathalie Quintane, Grand ensemble, Paris, P.O.L, 2008, p. 102-103; dorénavant GE. L'autrice souligne.

44. À ce sujet, voir DN 226-232.

\section{RÉSUMÉS}

À rebours d'une hypothèse répandue qui fait de Tomates (2010) un point tournant de la production de Nathalie Quintane, cet article insiste sur les similitudes qui unissent la «première" et la «seconde» portion de son œuvre. Je montrerai que plusieurs des logiques auxquelles répondent les plus récentes parutions de l'écrivaine trouvent leurs fondements dans ses premiers textes : loin de mener d'une part un travail d'expérimentation formelle et de l'autre un travail de thématisation d'enjeux politiques, Quintane problématise des représentations communément partagées depuis «Ah » (1996), Chaussure (1997) et Grand ensemble (2008) jusqu'à Tomates, "Stand up » (2014) et Un œil en moins (2018). En plus de mettre au jour la grande cohérence de l'œuvre, l'étude des figures du pouvoir qui peuplent ces différents textes permettra de décrire deux des procédés par lesquels l'écrivaine s'attaque, dans sa pratique, aux imaginaires qui fondent l'autorité des puissant·es. Qu'elle élabore des portraits caricaturaux ou qu'elle détourne des discours, Quintane s'applique moins à critiquer explicitement les politicien'ne's convoquée's qu'à faire saillir, par un jeu de loupe, les failles qui fragilisent leur stature ou leur rhétorique.

\section{INDEX}

Mots-clés : politique de la littérature, figures du pouvoir, ironie, détournement 


\section{AUTEURS}

\section{MARC-ANTOINE BLAIS}

Université du Québec à Montréal 